\title{
A NOTE CONCERNING THE IDEAL OF NUCLEAR OPERATORS
}

\author{
by BRUCE A. BARNES
}

(Received 11 January, 1995)

Abstract. Let $\mathscr{A}$ be a Banach algebra of bounded linear operators such that $\mathscr{A}$ contains every operator with finite dimensional range. Then $\mathscr{A}$ contains every nuclear operator.

1. Introduction. Let $X$ be an infinite dimensional Banach space. We adopt the following notation for the various spaces of operators involved here:

$$
\begin{aligned}
& \mathscr{B}(X) \equiv \text { the algebra of all bounded linear operators on } X ; \\
& \mathscr{F}(X) \equiv \text { the ideal of finite rank operators in } \mathscr{B}(X) ; \\
& \mathcal{N}(X) \equiv \text { the ideal of nuclear operators in } \mathscr{B}(X) .
\end{aligned}
$$

Also, let $\|\cdot\|_{o p}$ denote the usual operator norm, and let $\|\cdot\|_{1}$ denote the usual natural complete algebra norm on $\mathcal{N}(X)$. A useful discussion of algebras of operators and of $\mathcal{N}(X)$ is given in $[2, \S 1.7]$.

The main purpose of this note is to prove the following result.

THEOREM 1. Let $\left(\mathscr{A},\|\cdot\|_{\mathscr{A}}\right)$ be a Banach algebra of operators with

$$
\mathscr{F}(X) \subseteq \mathscr{A} \subseteq \mathscr{B}(X) \text {. }
$$

Then $\mathcal{N}(X) \subseteq \mathscr{A}$, and furthermore, there exists $M>0$ such that for all $T \in \mathcal{N}(X)$, $\|T\|_{\mathscr{A}} \leq M\|T\|_{1}$.

As a consequence of the theorem, $\mathcal{N}(X)$ is the smallest Banach algebra of operators that contains $\mathscr{F}(\mathrm{X})$. It has long been noted that $\mathcal{N}(\mathrm{X})$ is the smallest non-zero Banach ideal of operators in $\mathscr{B}(\mathrm{X})$.

A natural example of an algebra of operators to which Theorem 1 applies occurs in the theory of linear operators on a real or complex Banach lattice $X$. A good brief introduction to the algebras of operators involved can be found in W. Arendt's paper [1]. We use the terminology from this paper. Let $\mathscr{B}^{r}(X)$ be the algebra of all regular operators on the Banach lattice $X . \mathscr{B}^{r}(X)$ is a Banach algebra in the $r$-norm, and it is known that $\mathscr{F}(X) \subseteq \mathscr{B}^{r}(X)$. The closure of $\mathscr{F}(X)$ in the $r$-norm is the Banach algebra of all $r$-compact operators, denoted by $\mathscr{K}^{r}(X)$. Applying Theorem 1 , we have $\mathcal{N}(X) \subseteq$ $\mathscr{K}^{r}(X)$.

2. Results. Before proving Theorem 1 , we deal with some preliminary results. For $x \in X$ and $\alpha \in X^{\prime}$ (the dual space of $X$ ), let $\alpha \otimes x$ be the operators in $\mathscr{F}(X)$ given by

$$
(\alpha \otimes x)(y)=\alpha(y) x \quad(y \in X) ;
$$

for $\alpha \in X^{\prime}\{\{0\}$, let $\alpha \otimes X$ denote the space

$$
\alpha \otimes X=\{\alpha \otimes x: x \in X\} .
$$

Glasgow Math. J. 38 (1996) 233-236. 
Then $\alpha \otimes X$ is a minimal left ideal of $\mathscr{F}(X)$. The minimal right ideals of $\mathscr{F}(X)$ have the form

$$
X^{\prime} \otimes x=\left\{\alpha \otimes x: \alpha \in X^{\prime}\right\},
$$

where $x \in X \backslash\{0\}$. An algebra norm on $\mathscr{F}(X)$ is complete on minimal left and right ideals when the spaces $\alpha \otimes X$ and $X^{\prime} \otimes X$ (as above) are all complete with respect to the norm.

In what follows we use the notation $\|\cdot\|$ for both the norm on $X$ and the norm on $X^{\prime}$.

Proposition 2. Let $|\cdot|$ be an algebra norm on $\mathscr{F}(X)$ that is complete on minimal left and right ideals. Then there exist constants $m>0$ and $M>0$ such that

$$
m\|\alpha\|\|x\| \leq|\alpha \otimes x| \leq M\|\alpha\|\|x\|,
$$

for all $x \in X$ and $\alpha \in X^{\prime}$.

Proof. By [3, Theorem (2.4.17), p. 69], there exists $m>0$ such that for all $x \in X$ and $\alpha \in X^{\prime}$ we have

$$
m\|\alpha\|\|x\|=m\|\alpha \otimes x\|_{o p} \leq|\alpha \otimes x| .
$$

Fix $\alpha \in X^{\prime} \backslash\{0\}$, and consider the minimal left ideal $\alpha \otimes X$. By hypothesis, $|\cdot|$ is complete on $\alpha \otimes X$. Also, clearly $\|\cdot\|_{o p}$ is complete on $\alpha \otimes X$ and so, by the open mapping theorem and (1), there exists $J_{\alpha}>0$ such that

$$
J_{\alpha}\|\alpha\|\|x\|=J_{\alpha}\|\alpha \otimes x\|_{o p} \geq|\alpha \otimes x|, \text { for all } x \in X .
$$

The same argument applied to minimal right ideals implies that for each $x \in X \backslash\{0\}$ there exists $K_{x}>0$ such that

$$
K_{x}\|\alpha\|\|x\|=K_{x}\|\alpha \otimes x\|_{o p} \geq|\alpha \otimes x|, \text { for all } \alpha \in X^{\prime} .
$$

For any $\alpha \in X^{\prime}$ define $\varphi_{\alpha}: X \rightarrow(\mathscr{F}(X),|\cdot|)$ by $\varphi_{\alpha}(x)=\alpha \otimes x$. By (2) $\varphi_{\alpha}$ is continuous for each $\alpha$. Let $\mathscr{C}$ be the collection

$$
\mathscr{C} \equiv\left\{\varphi_{\alpha}: \alpha \in X^{\prime},\|\alpha\|=1\right\} .
$$

By (3) this collection of operators is pointwise bounded on $X$. Thus, by the uniform boundedness theorem, there exists $M>0$ such that $\left\|\varphi_{\alpha}\right\|_{o p} \leq M$, for all $\alpha \in X^{\prime}$ with $\|\alpha\|=1$. Therefore

$$
|\alpha \otimes x|=\left|\varphi_{\alpha}(x)\right| \leq M\|x\|\|\alpha\|, \text { for all } x \in X \text { and } \alpha \in X^{\prime} .
$$

Any operator $F \in \mathscr{F}(X)$ can be written in the form $F=\sum_{k=1}^{n} \alpha_{k} \otimes x_{k}$, where $\left\{x_{k}\right\} \subseteq X$ and $\left\{\alpha_{k}\right\} \subseteq X^{\prime}$. The projective tensor norm on $\mathscr{F}(X)$, denoted by $\|\cdot\|_{p}$, is defined by

see [2, p. 99].

$$
\|F\|_{p} \equiv \inf \left\{\sum_{k=1}^{n}\left\|\alpha_{k}\right\|\left\|x_{k}\right\|: F=\sum_{k=1}^{n} \alpha_{k} \otimes x_{k}\right\}
$$

COROLLARY 3. Let $|\cdot|$ be an algebra norm on $\mathscr{F}(X)$ that is complete on minimal left and right ideals. Then there exist $m>0$ and $M>0$ such that for all $F \in \mathscr{F}(X)$, we have

$$
m\|F\|_{o p} \leq|F| \leq M\|F\|_{p} .
$$


Proof. Again, the existence of $m>0$ for which $m\|F\|_{o p} \leq|F|$, for all $F \in \mathscr{F}(X)$, follows from [3, Theorem (2.4.17)]. Now let $M>0$ be as in the statement of Proposition 2. If $F \in \mathscr{F}(X)$ with $F=\sum_{k=1}^{n} \alpha_{k} \otimes x_{k}$, then

$$
|F| \leq \sum_{k=1}^{n}\left|\alpha_{k} \otimes x_{k}\right| \leq M\left(\sum_{k=1}^{n}\left\|\alpha_{k}\right\|\left\|x_{k}\right\|\right) .
$$

It follows that $|F| \leq M\|F\|_{p}$.

At this point we remind the reader of the definition of nuclear operator (following [2, p. 98]). An operator $T \in \mathscr{B}(X)$ is nuclear if there exist sequences $\left\{x_{k}\right\} \subseteq X$ and $\left\{\alpha_{k}\right\} \subseteq X^{\prime}$ satisfying

$$
\sum_{k=1}^{\infty}\left\|\alpha_{k}\right\|\left\|x_{k}\right\|<\infty \text { and } T(x)=\sum_{k=1}^{\infty} \alpha_{k}(x) x_{k},
$$

for all $x \in X$. The natural norm on $\mathcal{N}(X)$ is

$$
\|T\|_{1}=\inf \left\{\sum_{k=1}^{\infty}\left\|\alpha_{k}\right\|\left\|x_{k}\right\|: T \text { is represented by } \sum_{k=1}^{\infty} \alpha \otimes x_{k} \text { (as above) }\right\} .
$$

The proof of Theorem 1. Let $\left(\not A,\|\cdot\|_{\mathscr{A}}\right)$ be as in the statement of Theorem 1. Assume that $\alpha \in X^{\prime} \backslash\{0\}$. Choose $y \in X$ with $\alpha(y)=1$. Then $E=\alpha \otimes y$ satisfies $E^{2}=E$, and $\mathscr{A} E=\alpha \otimes X$. Since $\mathscr{A} E$ is a closed, and hence complete, subspace of $\mathscr{A}$, we have $\|\cdot\|_{\mathscr{A}}$ is complete on minimal left ideals of $\mathscr{F}(X)$. A similar argument shows that $\|\cdot\|_{\mathscr{A}}$ is complete on minimal right ideals of $\mathscr{F}(X)$. Therefore Proposition 2 applies.

Now let $T \in \mathcal{N}(X)$, so that there exist $\left\{x_{k}\right\} \subseteq X$ and $\left\{\alpha_{k}\right\} \subseteq X^{\prime}$ with $\sum_{k=1}^{\infty}\left\|\alpha_{k}\right\|\left\|x_{k}\right\|<\infty$, and $T(x)=\sum_{k=1}^{\infty} \alpha_{k}(x) x_{k}$, for all $x \in X$. Let $M$ be as in Proposition 2. Set $S_{n}=\sum_{k=1}^{n} \alpha_{k} \otimes x_{k}$. For $m>n$, we have

$$
\begin{aligned}
\left\|S_{m}-S_{n}\right\|_{\mathscr{A}} & \leq \sum_{k=n+1}^{m}\left\|\alpha_{k} \otimes x_{k}\right\|_{\mathscr{A}} \\
& \leq M\left(\sum_{k=n+1}^{m}\left\|\alpha_{k}\right\|\left\|x_{k}\right\|\right) \rightarrow 0 \text { as } m>n \rightarrow \infty .
\end{aligned}
$$

It follows that there exists $S \in \mathscr{A}$ with $\left\|S-S_{n}\right\|_{\mathscr{A}} \rightarrow 0$. Also, by [3, Theorem (2.4.17)], the $\mathscr{A}$-norm dominates the operator norm, and so $\left\|S-S_{n}\right\|_{o p} \rightarrow 0$. This implies that $T=S \in \mathscr{A}$. Also,

$$
\begin{aligned}
\|T\|_{\mathscr{A}} & =\lim _{n \rightarrow \infty}\left\|S_{n}\right\|_{\mathscr{A}} \leq \limsup _{n \rightarrow \infty}\left(\sum_{k=1}^{n}\left\|\alpha_{k} \otimes x_{k}\right\|_{\mathscr{A}}\right) \\
& \leq \limsup _{n \rightarrow \infty} M\left(\sum_{k=1}^{n}\left\|\alpha_{k}\right\|\left\|x_{k}\right\|\right)=M \sum_{k=1}^{\infty}\left\|\alpha_{k}\right\|\left\|x_{k}\right\| .
\end{aligned}
$$

Thus, $\|T\|_{\mathscr{A}} \leq M\|T\|_{1}$.

Finally, we present two more applications of Theorem 1. The following result is 
essentially Theorem (2.8.21) in C. Rickart's book [3]. The conclusion of the result has been strengthened by a direct (and obvious) application of Theorem 1.

Corollary 4. Let $|\cdot|$ be an algebra norm on $\mathscr{F}(X)$ and let $\mathscr{A}$ be the completion of $\mathscr{F}(X)$ with respect to this norm. Then there exists a representation $a \rightarrow T_{a}$ of $\mathscr{A}$ on $X$ whose kernel is the radical of $\mathscr{A}$ and such that each element of $\mathscr{F}(X)$ maps into itself. Furthermore, the image $\left\{T_{a}: a \in \mathscr{A}\right\}$ contains $\mathcal{N}(X)$.

When $H$ is a HIlbert space, two of the most important ideals in $\mathscr{B}(H)$ are $\mathscr{C}_{2}(H)$, the Hilbert-Schmidt operators on $H$, and $\mathscr{C}_{1}(\mathrm{H})$, the trace class operators on $H$; see R. Schatten [4]. Of course $\mathscr{C}_{1}(H)=\mathcal{N}(H)$. As is well-known (sometimes by definition), if $T, S \in \mathscr{C}_{2}(H)$, then $T S \in \mathscr{C}_{1}(H)$. Theorem 1 has the following amusing corollary in this context.

COROLlARY 5. Let $\mathscr{A}$ be a Banach algebra of operators on $H$ with

$$
\mathscr{F}(H) \subseteq \mathscr{A} \subseteq \mathscr{C}_{2}(H) \text {. }
$$

Then $\mathscr{A}$ is an ideal in $\mathscr{C}_{2}(H)$.

\section{REFERENCES}

1. W. Arendt, On the $o$-spectrum of regular operators and the spectrum of measures, Math. $Z$. 178 (1981), 271-278.

2. T. Palmer, Banach algebras and the general theory of *-algebras in Vol. I, Encyclopedia of Math. and its Applications (Cambridge University Press, 1994) 49.

3. C. Rickart, Banach Algebras (D. Van Nostrand Co., 1960).

4. R. Schatten, Norm ideals of completely continuous operators (Springer-Verlag, 1960).

Department of Mathematics

UNIVERSITY OF OREGON

EUGENE, OREGON 97403

U.S.A. 